\title{
BMJ Open Multiple treatment comparison of seven new drugs for patients with advanced malignant melanoma: a systematic review and health economic decision model in a Norwegian setting
}

\author{
Eva Pike, ${ }^{1}$ Vida Hamidi, ${ }^{1}$ Ingvil Saeterdal, ${ }^{1}$ Jan Odgaard-Jensen, ${ }^{1}$ \\ Marianne Klemp ${ }^{1,2}$
}

To cite: Pike E, Hamidi V, Saeterdal I, et al. Multiple treatment comparison of seven new drugs for patients with advanced malignant melanoma: a systematic review and health economic decision model in a Norwegian setting. BMJ Open 2017;7:e014880. doi:10.1136/ bmjopen-2016-014880

- Prepublication history and additional material for this paper are available online. To view these files please visit the journal online (http://dx.doi. org/10.1136/bmjopen-2016014880).

Received 24 0ctober 2016 Revised 31 May 2017 Accepted 3 July 2017

CrossMark

${ }^{1}$ Norwegian Institute of Public Health, Oslo, Norway

${ }^{2}$ Department of Pharmacology, University of Oslo, Oslo, Norway

Correspondence to

Dr Marianne Klemp;

Marianne.Klemp@fhi.no

\section{ABSTRACT}

Objective To assess the relative effectiveness and costeffectiveness of seven new drugs (cobimetinib, dabrafenib, ipilimumab, nivolumab, pembrolizumab, trametinib and vemurafenib) used for treatment of patients with advanced malignant melanoma in the Norwegian setting.

Design A multiple technology assessment.

Patients Patients with advanced malignant melanoma aged 18 or older.

Data sources A systematic search for randomised controlled trials in relevant bibliographic databases. Methods We performed network meta-analyses using both direct and indirect evidence with dacarbazine as a common comparator. We ranked the different treatments in terms of their likelihood of leading to the best results for each endpoint. The cost-utility analysis was based on a probabilistic discrete-time Markov cohort model. The model calculated the costs and quality-adjusted life years (QALYs) with different treatment strategies from a healthcare perspective. Sensitivity analysis was performed by means of Monte Carlo simulation.

Results Monotherapies with a programmed cell death 1 (PD-1) immune-checkpoint-inhibitor had a higher probability of good performance for overall survival than monotherapies with ipilimumab or BRAF/MEK inhibitors. The combination treatments had all similar levels of effectiveness to the PD-1 immune-checkpointinhibitors. PD-1 immune-checkpoint-inhibitors are more effective and more costly compared with ipilimumab in monotherapy. Nivolumab in combination with ipilimumab had higher costs and the same level of effectiveness as the PD-1 immune-checkpoint-inhibitors in monotherapy. BRAF/MEK inhibitor combinations (dabrafenib and trametinib or vemurafenib and cobimetinib) had both similar effectiveness and costeffectiveness; however, the combination therapies are more likely to give higher quality adjusted life year gains than BRAF or MEK inhibitor monotherapies, but to a higher cost.

Conclusions None of the drugs investigated can be considered cost-effective at what has normally been considered a reasonable willingness-to-pay (WTP) in Norway. Price reductions (from the official list prices) in the
Strengths and limitations of this study

- To our knowledge, a relative comparison of the different new drugs used for patients with advanced malignant melanoma has not been done by any other study, neither for effectiveness nor for costeffectiveness.

- Important differences between the drugs regarding patients' health-related quality of life (HRQoL) may not have been captured in our analysis, due to very limited evidence on $\mathrm{HRQ} \mathrm{OL}$ for the alternative interventions.

- There is uncertainty with regard to the correct treatment duration in the model, both for the new immunotherapies and the BRAF/MEK inhibitors.

- There is lack of documentation regarding the longterm effect of the newer drugs. Further research could change current estimates and consequently the health economic results.

region of $63 \%-84 \%$ would be necessary for these drugs to be cost-effective at a WTP of $€ 55850$ per QALY.

\section{INTRODUCTION}

Malignant melanoma is the most serious form of skin cancer. ${ }^{1}$ These tumours originate in the pigment-producing melanocytes in the basal layer of the epidermis. ${ }^{2}$ Malignant melanoma is divided into four stages, where stage I is the least severe and stage IV the most severe. Stage III includes locally advanced (inoperable, regional disease) and stage IV includes distant metastasis. ${ }^{34}$ The incidence of malignant melanoma in Norway is among the highest in the world ${ }^{5}$ with 1719 new cases in 2013. ${ }^{6}$ Malignant melanoma is the cancer type that increases most in Norway. ${ }^{1}$ For persons aged between 15 and 49 years, this is the second most frequent cancer type for both sexes together. ${ }^{7}$ A family history of 
malignant melanoma may be present in $5 \%-10 \%$ of the melanoma cases. ${ }^{3}$

Surgery is the primary treatment for malignant melanoma and currently also the only potentially curative treatment. ${ }^{5}$ Early diagnosis and appropriate surgical treatment cures $80 \%-90 \%$ of patients, while $10 \%-20 \%$ experience a relapse as local/regional recurrence or distant spreading. ${ }^{5}$ Patients with metastatic malignant melanoma have poor prognosis. ${ }^{7}$ The 5 -year relative survival rate for distant melanoma (stage IV) for the period 2009-2013 in Norway was 12.3\% for men and $24.5 \%$ for women. ${ }^{6}$

Dacarbazine has been the standard drug treatment for most patients. ${ }^{5}$ However, such chemotherapy has low response rates and has not been demonstrated to be life-extending. ${ }^{5}$ Recently, several new drugs have been under development for the treatment of malignant melanoma. As a result of growing clinical experience with these new drugs, treatment of advanced malignant melanoma has changed in the last $2-3$ years. ${ }^{57}$

The new drugs have different mechanisms of action: (1) affect the immune system (ipilimumab, nivolumab and pembrolizumab $)^{8}$; (2) inhibitors of mutated BRAF (serine-threonine protein kinase B-RAF) (dabrafenib and vemurafenib) ${ }^{910}$ or (3) MEK inhibitors (inhibit the mitogen-activated protein kinase pathway) (cobimetinib and trametinib). ${ }^{11} 12$

The drugs acting on the immune system do so by blocking mechanisms that limit activation of $\mathrm{T}$ cells. Activated $\mathrm{T}$ cells can be limited by CTLA-4 (cytotoxic T-lymphocyte-associated protein), a coinhibitory molecule of the immune system and by programmed cell death 1 (PD-1) with its ligands PD-L1 and PD-L2, which is expressed in peripheral tissues and cancers. ${ }^{8}$ Ipilimumab acts by blocking CTLA-4, whereas nivolumab and pembrolizumab block the interaction of the PD-1 receptor with its two ligands PD-L1 and PD-L2. ${ }^{813}$

Forty to fifty per cent of the patients with metastatic malignant melanoma have activated mutations in serine-threonine protein kinase B-RAF (BRAF). ${ }^{7}$ This knowledge has led to the development of the drugs, dabrafenib and vemurafenib, which are BRAF inhibitors. The use of a MEK inhibitor (cobimetinib or trametinib) together with a BRAF inhibitor may reduce the resistance seen to single agent BRAF inhibitors. ${ }^{14}$ The MEK inhibitors can also be used as single therapies.

The Norwegian Medicines Agency (NOMA), Canadian Agency for Drugs and Technologies in Health (CADTH) and National Institute for Health and Clinical Excellence (NICE) have performed single technology assessments on drugs used for metastatic melanoma, such as dabrafenib, ${ }^{1516}$ ipilimumab, ${ }^{17-20}$ trametinib ${ }^{21}$ and vemurafenib. ${ }^{22}{ }^{23}$ However, none of these assessments compared the different new drugs for patients with advanced malignant melanoma relatively to each other. We have, therefore, conducted this multiple technology assessment including all new drugs for these patients in a Norwegian setting.

\section{METHODS}

\section{Literature search and selection of studies}

We included randomised controlled trials (RCTs) which examined overall survival (OS), progression-free survival (PFS), health-related quality of life (HRQoL) and serious adverse events (SAEs) in patients aged 18 or older with advanced malignant melanoma. We were interested in the following interventions: cobimetinib, dabrafenib, ipilimumab, nivolumab, pembrolizumab, trametinib and vemurafenib compared with placebo or any drug treatment. The drugs can be used as monotherapy or in combination with each other.

A systematic literature search was performed in February 2015 and updated in September 2015 and in May 2017. We limited the search to trials published in year 2000-2017 since the interventional drugs have entered the market recently. We searched the following databases: Ovid MEDLINE(R) In-Process and Other Non-Indexed Citations and Ovid MEDLINE(R) version 1946 to Present; Embase version 1974 to present; Cochrane Central Register of Controlled Trials (Central); Web of Science; PubMed (epub ahead of print). The complete search strategies are presented in online supplementary material 1. We also searched Google Scholar. The websites of selected health technology assessment agencies were searched in September 2015. We contacted the pharmaceutical companies that have marketing authorisation or represent the interventional drugs to obtain additional information as published articles, abstracts/posters that fulfil our inclusion criteria. We also checked for RCTs in the relevant systematic reviews, reviews or meta-analyses which we identified. We looked for ongoing trials in ClinicalTrials.gov and WHO International Clinical Trials Registry Platform in August 2015.

Two reviewers independently inspected all citations to identify potentially relevant articles based on title and/or abstract. Full text publications were obtained for articles appearing to meet our inclusion criteria. Two persons independently assessed whether the article was relevant or not according to our list of inclusion criteria. Disagreements were resolved by discussion or by consulting a third reviewer. One reviewer extracted data from the included articles and another reviewer checked these results for accuracy.

\section{Statistical analyses and presentation of results}

We conducted a systematic review and meta-analyses of existing literature, following the methods recommended in the PRISMA statement for reporting systematic reviews and meta-analyses of studies that evaluate healthcare interventions. ${ }^{24}$ The protocol for the systematic review can be seen in online supplementary material 2 .

We expressed the comparative effectiveness of the treatments as the relative risk (RR) of dichotomous endpoints, HR for time-to-event endpoints and mean difference (MD) for continuous endpoints. If a continuous outcome had been measured/reported using different instruments/scales in the included RCTs, we 
would have calculated the standardised mean difference (SMD). For all endpoints 95\% CIs (results from pairwise meta-analyses) or credible intervals (CrI, results from network meta-analyses) were calculated for the RR, HR, MD or SMD. The CrI is the Bayesian analogue to the CIs used in traditional frequentist statistical approaches. We considered a result 'significant' if the CrI did not include $\mathrm{RR} / \mathrm{HR}=1$ or $\mathrm{MD} / \mathrm{SMD}=0$.

We performed a network meta-analysis (NMA) for each clinical endpoint individually. We did this by combining direct and indirect effects of the interventions of interest for each endpoint. The analysis was based on multiple treatments meta-analysis as described by Salanti. ${ }^{25}$ We used the arm-based network meta-analysis method (a Bayesian method based on Markov Chain Monte Carlo simulation). All NMAs were performed using Winbugs version 1.4.3 (Imperial College and MRC, UK). The statistical analysis was based on binomial likelihoods (dichotomous endpoints) and normal likelihood (continuous endpoints), with vague priors for the trial baselines, basic parameters (normal distribution with mean 0 and SD 0.0001 ) and the random effects SD (uniformly distributed in the interval 0-2) and takes the correlation structure induced by multi arm trials into account. For time-toevent endpoints (OS and PFS), with HR as the measure of effect, we used the method described by Woods $e t a l^{26}$ to combine HRs, cumulative number of events and median survival statistics. We used a random effects model. We checked for incoherence between direct and indirect evidence by 'node-splitting'. ${ }^{27}$ We calculated the direct and indirect estimates of effect and the corresponding Bayesian ' $p$ values' for incoherence.

We also ranked the different treatments in terms of their likelihood of leading to the best results for each endpoint. We based the rankings on the surface under the cumulative ranking curve (SUCRA) ${ }^{28}$ We interpreted the rankings cautiously taking into account the quality of the evidence.

The dose-comparison trials of Hamid $e t a l^{29}$ and Robert et $a l^{30}$ showed that the effect of ipilimumab and pembrolizumab did not seem to depend on the doses given. As a consequence, we have combined different doses of ipilimumab and pembrolizumab into one group. We have treated different doses of the other interventions in a similar way; this was however, only relevant for trametinib.

For the endpoint PFS, we assumed that participants who dropped out experienced disease progression if a HR between intervention and control was not reported. For all other endpoints, we did not perform imputations for missing data. We based the statistical analyses on the intention-to-treat principle (all participants analysed in the group to which they were allocated and all available data included in the analyses).

\section{Quality of the evidence}

The quality of the included studies was assessed using the Cochrane Collaboration's tool for Risk of Bias assessment. ${ }^{31}$ We assessed the overall quality of evidence for each endpoint using GRADE (Grading of Recommendations Assessment, Development and Evaluation). We followed the guidelines provided by the GRADE working group $^{32}$ and categorised our confidence in the effect estimates into four levels: high, moderate, low and very low. The quality of the direct evidence, indirect evidence and the combined evidence from the NMAs was evaluated using the GRADE approach for network meta-analyses. ${ }^{33}$ We used the Guideline Development Tool, ${ }^{34}$ while evaluating the quality of the direct evidence.

\section{Economic evaluation}

In order to assess the cost-effectiveness of the interventions targeting patients with metastatic and/or unrespectable malignant melanoma, we made a probabilistic decisionanalytic model.

The model has three mutually exclusive disease-related health states: (1) progression-free disease (PFS), (2) progressed disease (PD) and (3) death (see online supplementary material 3). All patients start in the progression-free disease state. For every model cycle, the arrows indicate that a proportion of the patients may remain in the same health state, another proportion may experience progression and another may die, determined by the transition probabilities.

Disease regression in the form of transition from progressed disease to PFS is not possible. The model does not include treatment sequences. The time horizon is 10 years with a monthly cycle-length. The analysis was carried out from a healthcare payer perspective. Costs and quality-adjusted life years (QALYs) were discounted at a rate of $4 \%$ per annum. We used $\mathrm{R}$ version 3.2.2 for the estimation of the cumulative density functions and baseline transition probabilities and TreeAge Pro 2015 for the decision modelling.

\section{Model parameters}

Table 1 shows the key parameters used in the base case analysis.

\section{Transition probabilities}

To estimate transition probabilities for the models, we first needed to determine the cumulative density functions for OS and PFS for control-arm. For the baseline OS and PFS, we fitted cumulative density functions from the dacarbazine arm of a RCT, published by Robert $e t a l^{35}$ and Maio et al. ${ }^{36}$ We used patient-level time to event data to construct Kaplan-Meier curves in these studies. The two trials by Robert $e t a l^{35}$ and Maio $e t a l^{36}$ have both only included patients that were not previously treated (table 1 in online supplementary material 4 ). The choice of cumulative density function was based on the best fit as evaluated by the Akaike information criterion.

We used the transition probability formula suggested by Briggs $e t a \hat{l}^{7}$ as the basis for calculating transition probability from alive to death (the transitions from health state 1 to 3 and from 2 to 3 ) and from PFS to progressed disease (1 to 2 ). From the fundamental relationships for 

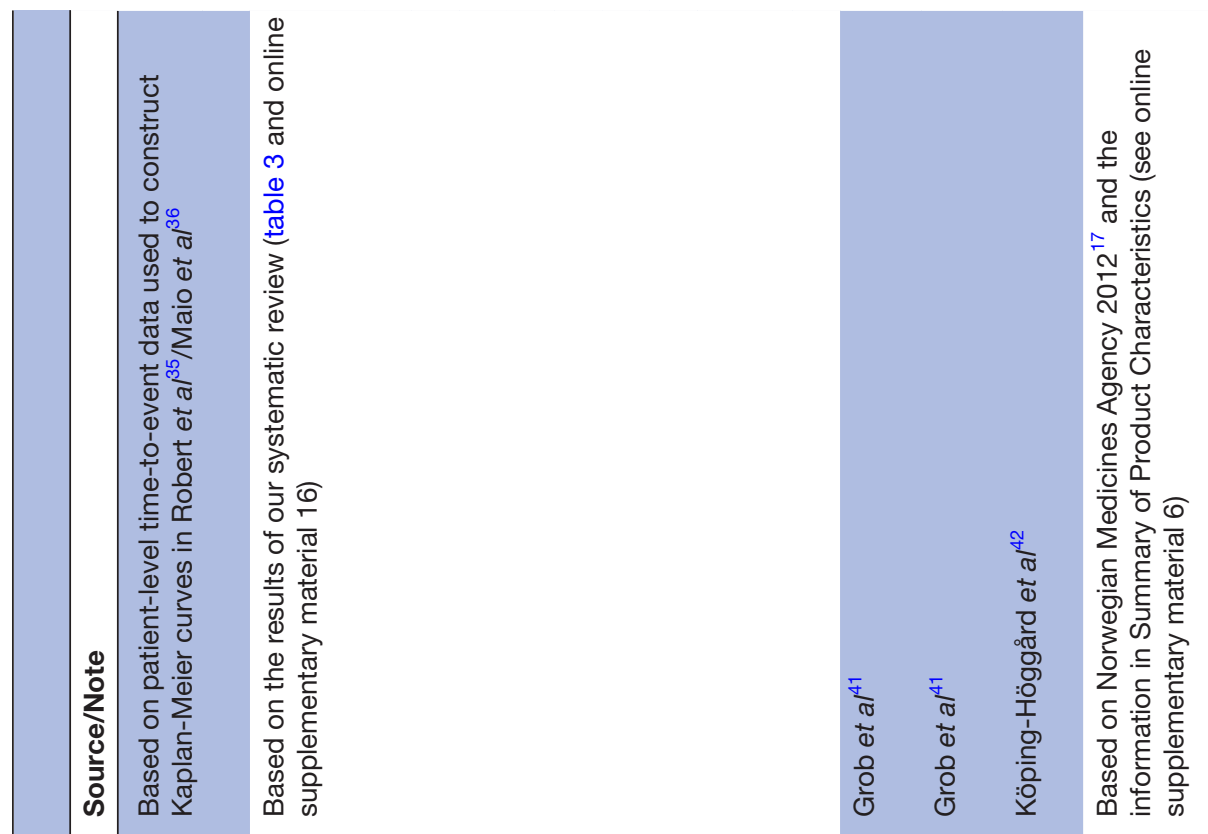

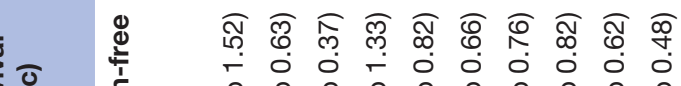

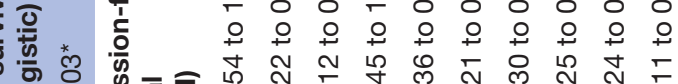

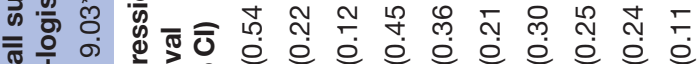

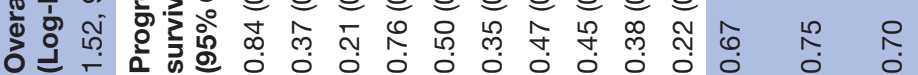

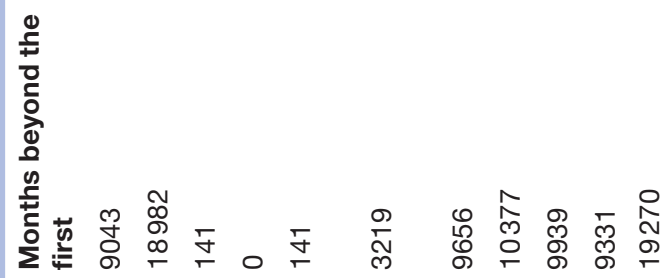

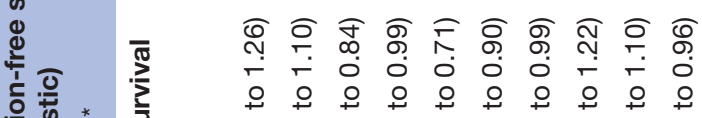

寸 की

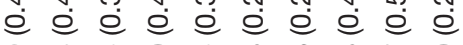
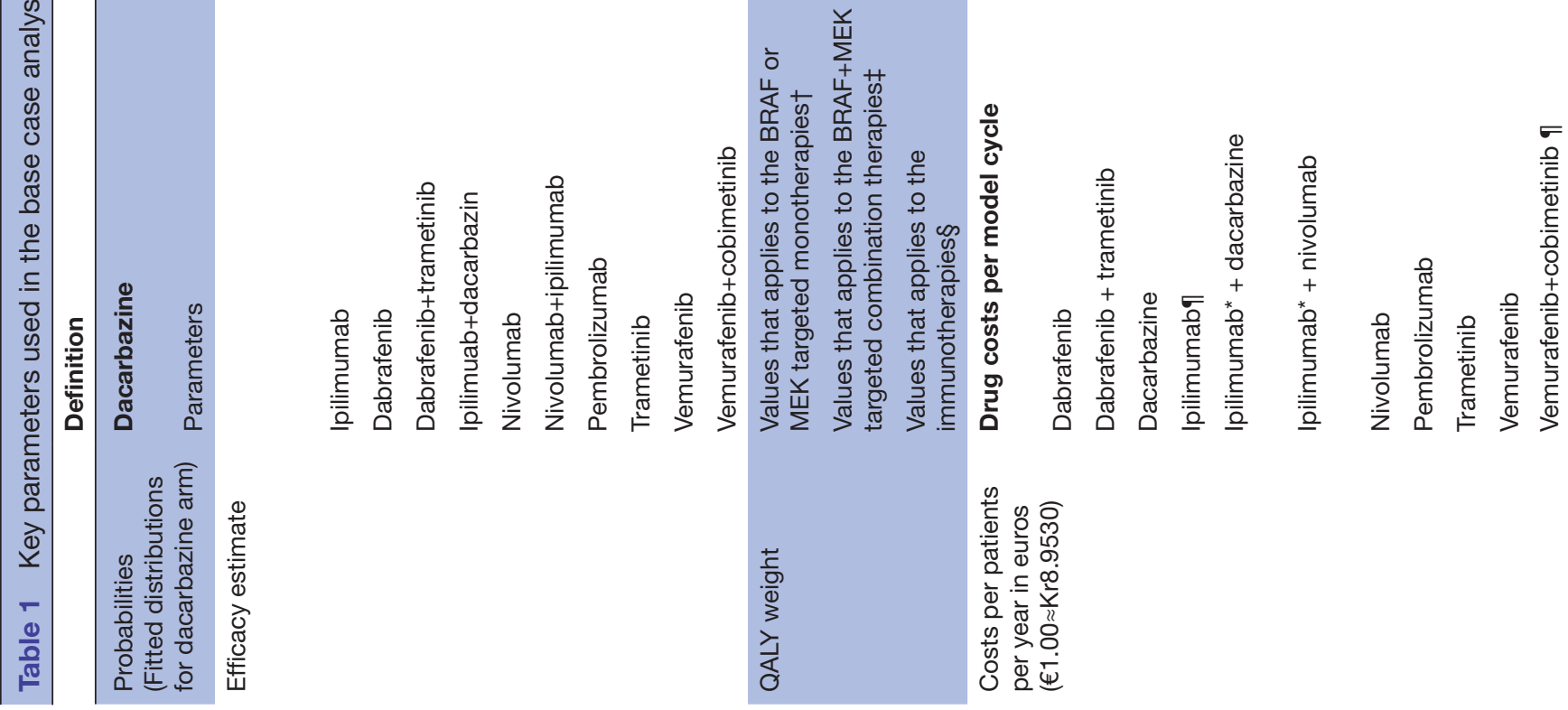


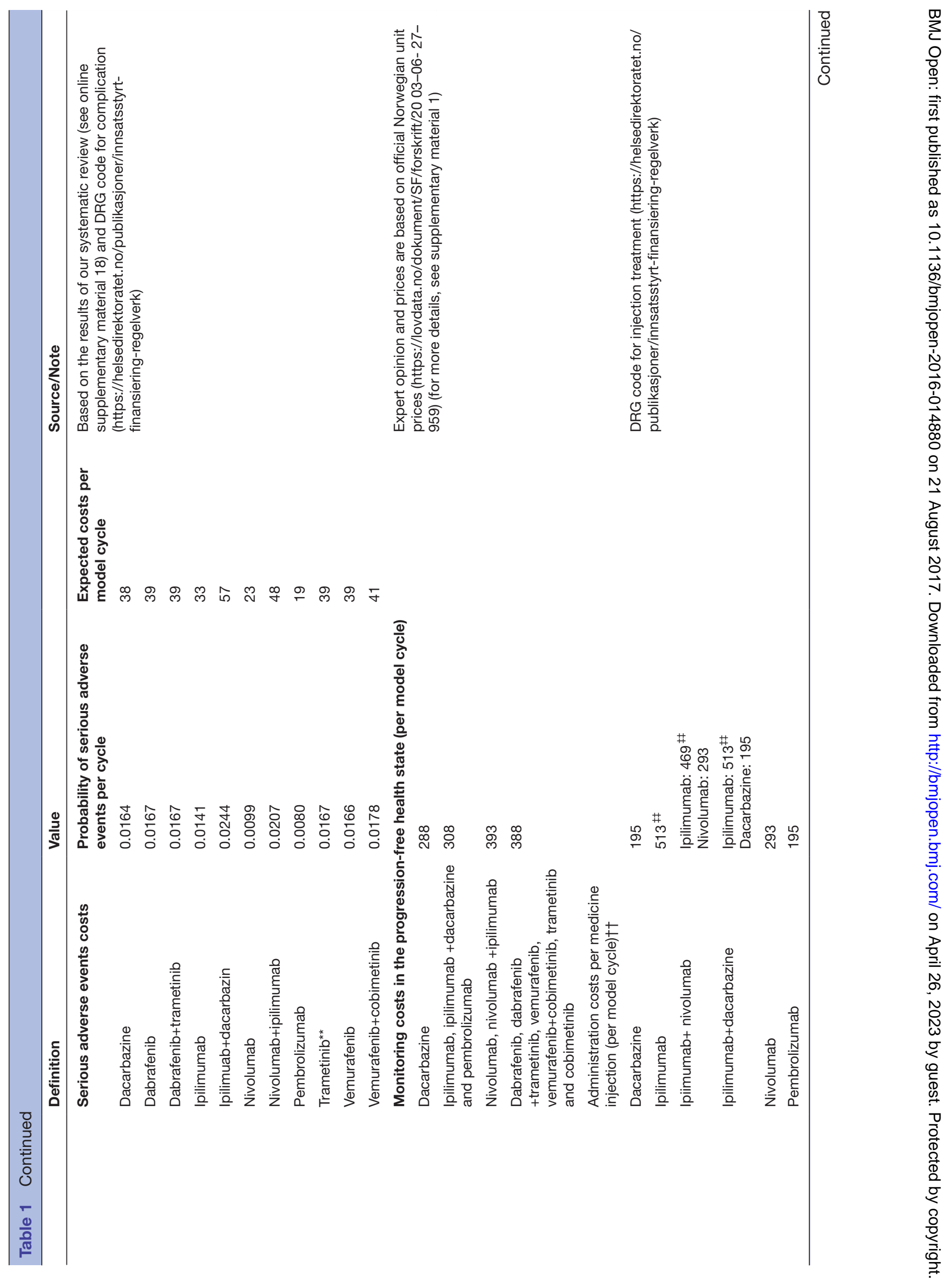


probabilities of mutually exclusive events, it follows that the transition probability from PFS to progressive disease ( 1 to 2 ) is one minus the probability of death minus the probability of staying in the PFS health state.

In practice, however, trial results report only the OS rate, which includes survival from both the progression-free and progressed disease states. Using the fundamental relationship described above can lead to double counting. To avoid this possibility, we calculated the transition probability from PFS to PD in two steps (see online supplementary material 3), first with regard to death (OS) and then for progression, conditional on having survived. Online supplementary material 5 gives more explanation for the choices made.

\section{Treatment effects}

We used the HRs relative to dacarbazine from the network meta-analysis (table 1) to adjust the baseline transitions probabilities.

In the base case analysis, the HRs are applied up to 2 years, assuming no treatment effects past 2 years of treatment for any of the interventions. Any accumulated survival and progression benefits would, however, have an impact also after treatment discontinuation.

\section{Costs}

All costs were expressed in 2015 Norwegian kroner and converted into Euros based on the average exchange rate for Norwegian kroner to Euros in 2015 (€1.00 Kr8.9530) reported by the Norwegian Central Bank. Table 1 summaries all costs included in the model.

BRAF gene mutation diagnostic testing

It has become current practice in Norway to test all patients with unresectable and/or metastatic malignant melanoma for the BRAF gene mutation. Information on the mutation status of the patient can be used to determine whether the patient should receive immunotherapy or BRAF gene mutation targeted therapy. In our model, the only treatment arm not tested for genetic mutations is the dacarbazine-arm. This arm was included as a comparator to reflect the situation as if the new therapies were not an option. In such a context, the results of the test would not add valuable information, as the treatment would be the same independently of the test result.

Based on expert's opinion, we assumed that all patients are tested with a BRAF qPCR test and around $15 \%$ with a BRAFpyro. The BRAF gene mutation rate among Norwegian patients with malignant melanoma is around $50 \%$. All patients who do not have the BRAF gene mutation are subsequently tested for neuroblastoma RAS viral oncogene homologue gene mutation. Our average cost estimate for the cost of testing is $€ 457$ per patient.

\section{Medicine costs}

The medicine costs depend on the acquisition price, the dosages and duration of treatment. Drug costs included in the model reflect the maximum pharmacy retail price, including VAT. We planned to conduct sensitivity analyses 
to determine the price at which each drug can be considered cost-effective. The dosages we used correspond to the information in the summary of Product Characteristics and an overview of the dosages used is provided in online supplementary material 6 .

At the time of writing, we did not have a maximum pharmacy retail price for cobimetinib. In the base case of the model, we chose to assume that cobimetinib has the same price as trametinib, that is, the combination cost was the sum of the monthly cost of vemurafenib in monotherapy plus trametinib, resulting in a combined monthly drug cost of about $€ 19270$ per month.

In clinical practice, the actual cumulative dose may, however, be lower than the planned cumulative dose, for instance due to drug intolerance. The relative dose intensity of dabrafenib in the Combi-D study was about $88 \%$ (personal communication). We used the same relative dose intensity for dabrafenib in combination with trametinib, trametinib, vemurafenib and vemurafenib in combination with cobimetinib in the model, based on expert advice.

Based on experience from clinical practice in Norway, the number of doses each patient receives on average with ipilimumab is likely to be less than 4 . From the relative dose intensity information reported in Postow et $a l^{38}$ we calculated a weighted average for ipilimumab in monotherapy of 3.5 doses and in combination therapy (with nivolumab) of 3.2 doses.

For the PD-1 immunotherapies, nivolumab and pembrolizumab, we were advised from our clinical experts to assume that treatment could be provided both in the progression-free and progressed health states. In addition, we assumed a gradual decline in the proportion being treated over time of those alive, according to information given in the appendix to Larkin $e t a l^{39}$ at 12 months of follow-up and assuming no treatment after 2 years.

There is no accumulation of medicine costs past 2 years due to the assumption that treatment stops at this time. In online supplementary material 7 , we have summarised all the assumptions we have made in the base case model.

In table 1, we have summarised the medicine costs estimates per model cycle (month), with the exception of ipilimumab, where the total drug cost is valid only for the first model cycle. For more details about the drug cost estimation and drug dosages, see online supplementary material 6.

\section{Drug dispensing costs}

We estimated that $80 \%$ of the patients receiving medicines in tablet form would prepare their doses alone, while the other $20 \%$ would get help from either a nurse (inpatients and homecare patients) or the hospital's pharmacy (outpatients). We assumed that the dispensing costs would be about $€ 46$ per month (based on expert opinion).

\section{Parenteral drugs administration costs}

Dacarbazine, ipilimumab, nivolumab and pembrolizumab are intravenous therapies, administered by a nurse who supervises the whole procedure. For all therapies given intravenously at hospital, we assumed that administration costs per dose were reflected by $7 \%$ of the reimbursement, that is, $€ 147$ (included wages, material and overhead costs).

\section{Drug-therapy related SAEs}

We chose to include SAEs requiring hospitalisation, that is, adverse events grade 3 and 4 . The monthly costs related to SAEs are determined by the cost of hospitalisation and the average monthly probability of such an event.

The costs of an adverse event-related hospitalisation were assumed to be equal to $100 \%$ of the reimbursement, that is, €2325. We estimated the average monthly rate of a SAE from patients included in the dacarbazine arm of the NCT00324155 trial published in Robert $e t a \hat{l}^{35},{ }^{13}$ the only publications without crossover after progression where in addition dacarbazine was administered as monotherapy. The average monthly rate of $1.64 \%$ is the arithmetic average of the monthly frequency of experiencing SAEs.

We adjusted the baseline risk of an adverse event with the RR of adverse event versus dacarbazine identified in our meta-analysis (see online supplementary material 8). Table 1 summarises the expected cost per model cycle for each treatment arm and the associated cycle probability.

Any serious treatment related adverse events post progression was assumed to be included in the monitoring cost for the progressed disease state.

\section{Monitoring costs}

In the progression-free health state, patients are followed up during and after treatment stop in order to assess the course of the disease. While in treatment, the intensity and content of this follow-up schedule varies across interventions and includes outpatient visits to specialists (oncologists and/or dermatologists), blood analyses and diagnostic imaging (CT, ultrasound, bone scintigraphy, PET and/or MR). The resulting cost estimates are shown in table 1. table 2.

In the progressed health state the costs consists of a mix of surgery, radiotherapy and palliative treatment at a hospital centre and/or through day care. Although we assume that some patients may be treated with PD-1 immunotherapy post progression, we consider a mix of best supportive care to best reflect the per cycle costs in the progressed health state.

See online supplementary material 6 for more details regarding our monitoring costs estimates.

\section{Health-related quality of life}

HRQoL data are based on the systematic search. We conducted a systematic search for published utility weights that were relevant for our model population and treatment options. For consistency, and noting that different utility instruments can yield different utility 
Table 2 Results when all incremental cost-effectiveness ratios refer to dacarbazine (time-horizon of 10 years)

\begin{tabular}{|c|c|c|c|c|c|}
\hline Interventions & Costs $(€)$ & Incremental cost & Effectiveness (QALYs) & Incremental effect & ICER (€/QALY) \\
\hline Dacarbazine* & 17995 & & 0.88 & & \\
\hline Trametinib† & 100708 & 82714 & 1.16 & 0.28 & 295405 \\
\hline Dabrafenib† & 105329 & 87334 & 1.23 & 0.35 & 249526 \\
\hline Vemurafenib† & 105394 & 87399 & 1.19 & 0.31 & 281932 \\
\hline Ipilimumab† & 106788 & 88793 & 1.36 & 0.48 & 184985 \\
\hline Ipilumab+dacarbazine† & 107071 & 89077 & 1.28 & 0.40 & 222692 \\
\hline Nivolumab* & 118792 & 100798 & 1.70 & 0.82 & 122924 \\
\hline Pembrolizumab† & 121325 & 103330 & 1.68 & 0.80 & 129162 \\
\hline Nivolumab+ipilimumab† & 168531 & 150537 & 1.69 & 0.81 & 185848 \\
\hline Vemurafenib+cobimetinib* & 276455 & 258460 & 1.77 & 0.89 & 290405 \\
\hline Dabrafenib+trametinib $†$ & 277648 & 259654 & 1.71 & 0.83 & 312836 \\
\hline
\end{tabular}

*Undominated.

†Dominated (including extended dominance).

QALY, quality-adjusted life year.

weights for the same health state, we focused on values based on EQ-5D, the most commonly used instrument. ${ }^{40}$

We used EQ-5D values from Grob et $a l^{41}$ for vemurafenib in monotherapy and dabrafenib and trametinib in combination therapy to inform the PFS and progressed disease health states for those interventions. Due to lack of data, these values were also used for dabrafenib monotherapy and the combination vemurafenib and cobimetinib.

Grob et $a t^{11}$ indicated a slight decrease in HRQoL following progression for vemurafenib in monotherapy, but an increase for the BRAF and MEK combination therapy. We chose not to include this increase in the analysis for the combination therapies, instead fixing the HRQoL at the same level as in the progressive free health state.

The EQ-5D values for the interventions involving immunotherapies are derived from a published single technology assessment of pembrolizumab compared with ipilimumab. $^{42}$

Table 1 shows the EQ-5D values we used in the model analysis for PFS and progressed disease. The immunotherapies have a slightly higher HRQoL in the PFS health states than the BRAF and MEK monotherapies and combination therapies, but the BRAF and MEK in combination therapies have a slightly higher HRQoL in the progressed disease state.

We used the BRAF or MEK monotherapies HRQoL values for the common comparator dacarbazine.

\section{Sensitivity analysis}

To explore the consequences of uncertainties in the base-case parameter values, we performed a probabilistic sensitivity analysis (PSA) in which all input parameters were randomly drawn from probability distributions and the model was run 10000 times. For probabilities and utility estimates, the beta-distributions were constructed on plausible ranges for parameters. For cost, we modelled gamma-distributions. Efficacy and safety parameters were assumed to have a log-normal distribution where mean and CIs from our network meta-analysis were used to estimate the parameters. All the model parameters and distributions are presented in online supplementary material 9. Our suggestions about cost-effectiveness will reflect a range of potential willingness-to-pay (WTP) values per gained QALY. Separate scenario analysis will investigate the importance of drug pricing, the choice of time horizon and HRQoL weights.

We also performed an analysis of the expected value of perfect information (EVPI) in order to explore the uncertainty surrounding specific groups of parameters and to show which groups of input parameters it is most useful to conduct further research. The EVPI for the parameter or group of parameters is the difference between the expected net benefit with perfect (additional) information and the expected value with current information about the parameter(s). ${ }^{37}$

\section{RESULTS \\ Description of included studies}

We identified 704 citations, additionally two citations were identified through manual search and 75 citations were received from the manufactures. Finally, 40 publications (24 full text publications and 16 abstracts/posters) met the prespecified inclusion criteria (figure 1). Those publications represent 17 unique clinical trials. The excluded publications, including reasons for the exclusions, are given in online supplementary material 10 . Our searches in websites of sister health technology assessment agencies in August 2015 did not identify further trials; for details, see online supplementary material 1. Possible relevant ongoing trials are listed in online supplementary material 1.

All the 17 RCTs were mostly of phase III, three trials were phase $\mathrm{II}^{43-45}$ and one was phase $\mathrm{I}^{46}$ Most of the trials 


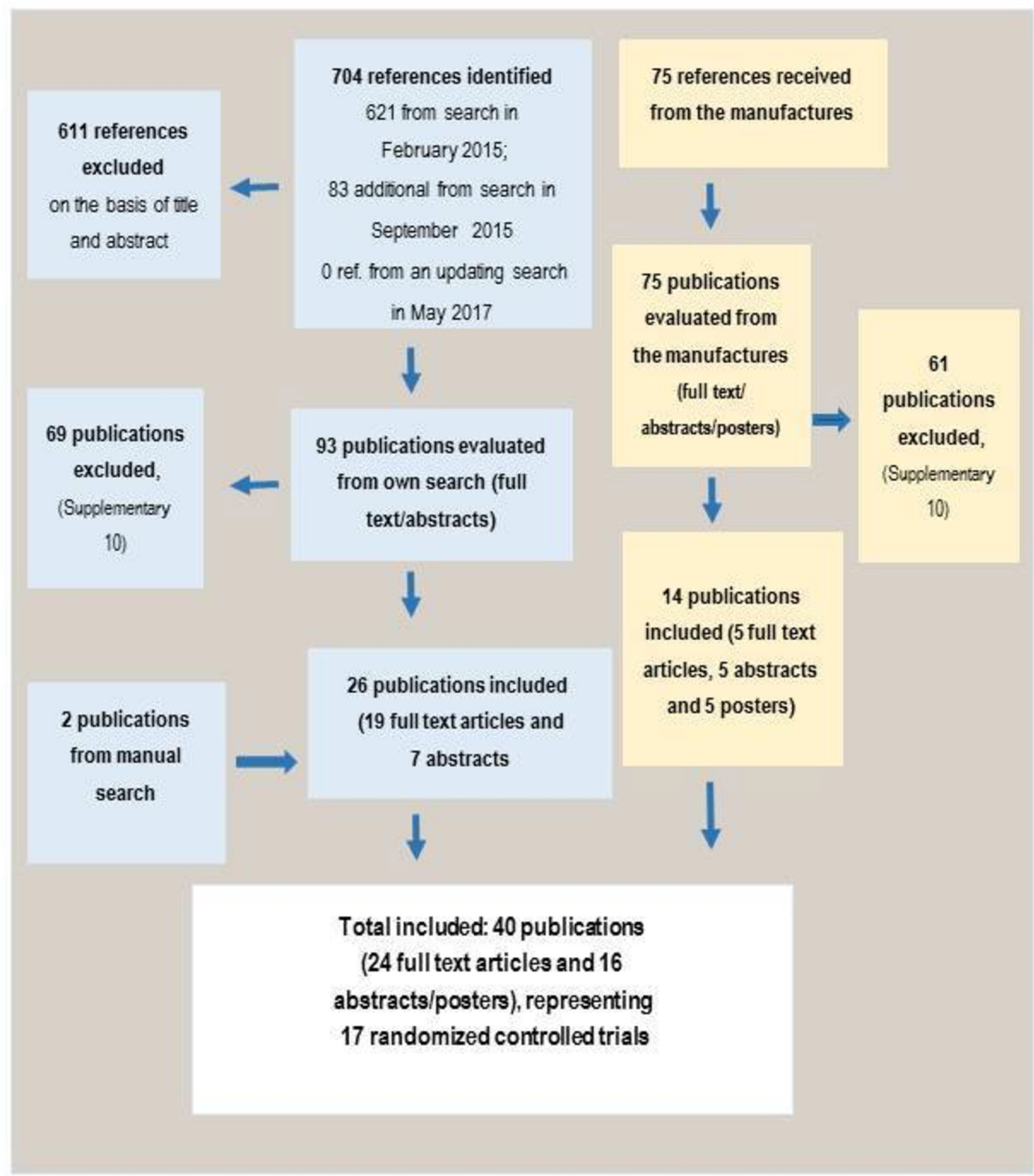

Figure 1 Flowchart of identification and selection of documentation.

(10-17) were open-labelled, the other seven trials were double-blinded. ${ }^{11} 13143547-49$ All were multicentre trials, and the majority were performed in North America and Europe. A total of 7482 patients were included in the 17 trials, with a range from 59 to 945 patients in the respectively trials. The 17 trials $(9-15,37-46)$ were published from 2010 to 2015 in a total of 40 publications. ${ }^{8-1435364143-71}$
Most of the publications were of new date, with 24 of the 40 publications published from 2014 and later. An overview of the included RCTs is presented in online supplementary material 4.

The trials included patients $\geq 18$ years, with advanced malignant melanoma. The median age ranged from 49 to 67 , and the proportion of males ranged from $49 \%$ 
to $74 \%$. The majority of the trials (10 of 17 ) included patients previously pharmacologically untreated. Four of the trials included both previously treated and untreated patients ${ }^{8124369}$ and three of the trials included only previously pharmacologically treated patients. ${ }^{44} 472$

Patients with identified BRAF V600 mutation, either specifically identified as BRAF V600E ${ }^{9} 10$ or BRAF V600E or $\mathrm{V} 600 \mathrm{~K}^{11} 12144369$ were included in the seven trials with $\mathrm{BRAF}$ and/or MEK inhibitors as the intervention. Patients who were included in the trials where the intervention acts on the immune system, had not identified any BRAF mutation, except in four trials ${ }^{44} 48454$ that identified both patients with and without BRAF mutation.

More details can be seen in online supplementary materials 4 and 11 .

Seven of the trials allowed patients in the control group to cross over to the intervention group after progression. ${ }^{44} 454957636471$ McArthur et a ${ }^{63}$ reported results for OS and PFS both as censored at the time of crossover and without censoring at the time of crossover. In our analyses, we only included the data without censoring at the time of crossover, since this method was used in the other trials.

Dacarbazine was used as the comparator in seven of the trials, ${ }^{910} 1335446972$ including three trials were the control groups were the investigator choice of chemotherapy: dacarbazine or paclitaxel, ${ }^{69}$ dacarbazine or paclitaxelin combination with carboplatin, ${ }^{72}$ or dacarbazine as one of five investigator-choice chemotherapies. ${ }^{44} 72$ The other comparators used in the included trials were ipilimumab for six of the trials, ${ }^{85-49}$ nivolumab in one trial, ${ }^{48}$ dabrafenib in two trials ${ }^{14}$ and vemurafenib in two trials. ${ }^{1112}$

Since all the interventions, except cobimetinib, were compared with dacarbazine, directly or indirectly, this was used as our common comparator in our network meta-analyses. The three arms using investigator choice of chemotherapy ${ }^{44569}$ were considered to be equivalent to dacarbazine. Consequently, these three arms were included as dacarbazine arms in the statistical analyses.
OS was measured with a follow-up time from 5 months ${ }^{10}$ to 5 years. ${ }^{36}$ When one study reported from more than one follow-up time points, we choose to extract from the first report measuring 2 years survival (if available) as well as from the latest available data. In our network meta-analyses we use the 2 years data.

We assessed the risk of bias for the endpoints in the included RCTs to be from low to high risk, mostly of low risk of bias; for more details, see online supplementary material 11.

\section{Clinical effectiveness}

Our results for the relative comparisons of the included drugs or combinations of drugs are based on the network meta-analyses. For OS, PFS and SAEs all the interventions/treatment strategies were included in the network; hence, they could all be compared relative to dacarbazine. Due to insufficient data we could not perform a network meta-analysis for HRQoL. Below, we present results from all the comparisons with available direct evidence. Estimate HRs and quality ratings for direct and indirect comparisons based on the network meta-analysis analyses can be found in online supplementary materials 12-14 for the outcomes OS, PFS and SAEs, respectively.

The evidence network for OS is shown in figure 2. The results of the random effects network meta-analysis for the comparisons between the interventions and the common comparator dacarbazine are presented in table 3. Table 3 also shows a ranking of the included treatments using the SUCRA, and a grading of the evidence from the network meta-analyses is presented.

We found that nivolumab, pembrolizumab, nivolumab combined with ipilimumab, vemurafenib combined with cobimetinib and dabrafenib combined with trametinib all have better OS than dacarbazine. The ranking as measured by the SUCRA suggests that nivolumab, pembrolizumab, nivolumab combined with ipilimumab, vemurafenib combined with cobimetinib and dabrafenib combined with trametinib have a higher probability of

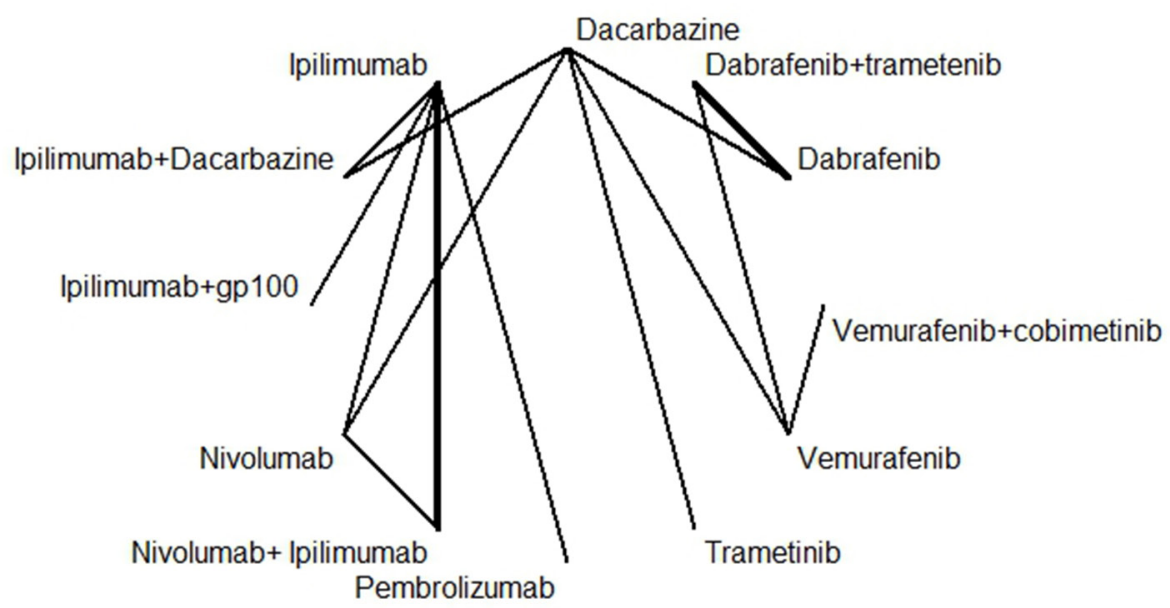

Figure 2 Evidence network for overall survival. 
Table 3 HRs for overall survival from network meta-analysis

\begin{tabular}{llll}
\hline Intervention & $\begin{array}{l}\text { HR relative to dacarbazine (Network } \\
\text { meta-analysis) }\end{array}$ & SUCRA & $\begin{array}{l}\text { Quality of evidence from the network } \\
\text { meta-analysis }\end{array}$ \\
\hline Nivolumab & $0.45(0.30$ to 0.71$)$ & 0.85 & Moderate \\
\hline Pembrolizumab & $0.46(0.26$ to 0.99$)$ & 0.81 & Very low \\
\hline Nivolumab+ipilimumab & $0.48(0.28$ to 0.90$)$ & 0.78 & Low \\
\hline Vemurafenib+cobimetinib & $0.50(0.26$ to 0.96$)$ & 0.73 & Moderate \\
\hline Dabrafenib+trametinib & $0.55(0.37$ to 0.84$)$ & 0.68 & Low \\
\hline Ipilimumab+dacarbazine & $0.70(0.47$ to 0.99$)$ & 0.41 & High \\
Ipilimumab & $0.69(0.44$ to 1.26$)$ & 0.40 & Very low \\
\hline Ipilimumab+gp100 & $0.72(0.40$ to 1.55$)$ & 0.36 & Very low \\
\hline Dabrafenib & $0.73(0.49$ to 1.10$)$ & 0.35 & Moderate \\
\hline Trametinib & $0.78(0.49$ to 1.22$)$ & 0.30 & Low \\
Vemurafenib & $0.77(0.54$ to 1.10$)$ & 0.29 & Moderate \\
Dacarbazine & 1 & 0.05 & - \\
\hline
\end{tabular}

SUCRA, surface under the cumulative ranking curve.

good performance than the other available treatment strategies (table 3).

The evidence network for PFS is shown in online supplementary material 15. In addition, online supplementary material 16 shows a ranking of the included treatments using the SUCRA together with the quality of evidence.

The ranking as measured by the SUCRA suggests that dabrafenib combination with trametinib and vemurafenib combined with cobimetinib has a higher probability of better performance for PFS than the other available treatment strategies (see online supplementary material 16).

The evidence network for SAEs is shown in online supplementary material 17.

In addition, online supplementary material 8 shows a ranking of the included treatments using the SUCRA together with the quality of evidence.

Based on the results of the network meta-analysis, we could not establish any differences between the available treatment strategies. However, the ranking as measured by the SUCRA suggests that pembrolizumab and nivolumab has a higher probability of fewer SAEs than the other available treatment strategies, even though we could not establish any differences (see online supplementary material 8).

The full network-meta-analysis results comparing all available treatment strategies for OS, PFS and SAEs are presented in online supplementary material 18.

More details about the estimates of OS, PFS and SAEs with quality rating (GRADE) for direct and indirect evidences are shown in the Summary of Finding tables in online supplementary material 19.

\section{Health-related quality of life}

Due to insufficient data, we have not performed network meta-analyses for HRQoL. Online supplementary material 20 presents a descriptive overview of the evidence for
HRQoL. More details of the studies are found in online supplementary material 11.

\section{Cost-effectiveness analysis}

The results of the incremental analysis are presented in table 2. Nivolumab and the combination of vemurafenib and cobimetinib were two undominated treatment strategies. Nivolumab had an incremental cost-effectiveness ratio against dacarbazine of about EUR

123000 per QALY gained. Vemurafenib in combination with cobimetinib had an incremental cost-effectiveness ratio against nivolumab of about $€ 2$ million per QALY gained.

Figure 3A shows the scatter plot for 10000 Monte Carlo simulations. From the top right corner, are the combinations dabrafenib in combination with trametinib and vemurafenib in combination with cobimetinib and the combination nivolumab and ipilimumab. The largest cloud of scatter consists of the BRAF inhibitors dabrafenib, vemurafenib and the MEK inhibitor trametinib, the immunotherapies ipilimumab, nivolumab and pembrolizumab.

The cost-effectiveness acceptability curves are presented in figure 3B. The results showed that nivolumab was the most cost-effective strategy for WTP values above $€ 123000$. However, both nivolumab and pembrolizumab had quite similar percentages of iterations being cost effective.

The results of EVPI indicated that the treatment efficacy data was the most influential source of uncertainty, followed by the HRQoL data, costs and SAEs data (HR for SAE) (the figure is presented in online supplementary material 21).

\section{Results for the immunotherapies}

Nivolumab was the undominated strategy and had an incremental effect of 0.82 QALYs and an incremental 


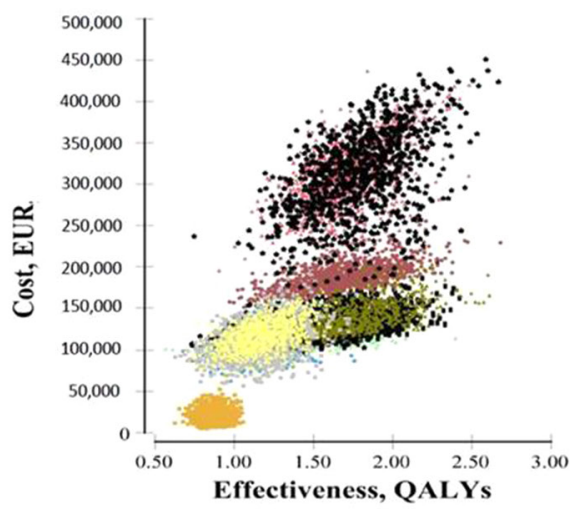

Figure 3 (A) Scatter plot for 10000 Monte Carlo simulations. dacarbazine. QALY, quality-adjusted life year.

cost-effectiveness ratio compared with dacarbazine of about $€ 123000$ per QALY gained.

When dacarbazine is excluded from the comparison nivolumab and pembrolizumab had very similar costs and effectiveness. The combination therapy nivolumab and ipilimumab had higher costs and the same level of effectiveness as nivolumab or pembrolizumab monotherapy. Online supplementary material 22 shows the incremental analysis when dacarbazine is excluded from the comparison.

When dacarbazine is included as a comparator (figure 3B), the PSA shows that nivolumab is more likely to be cost effective than the alternatives for WTP values exceeding about $€ 123000$. Pembrolizumab is however not far behind with respect to the percentage of iterations being cost-effective. When dacarbazine is excluded from the analysis (see online supplementary material 23), ipilimumab in monotherapy is more likely to be cost effective than the alternatives for WTP values below $€ 34000$, where after nivolumab is more likely to be cost effective than the alternatives for increasing WTP values.

\section{Results for the BRAF and MEK monotherapies and combination therapies}

Dabrafenib and vemurafenib in combination with cobimetinib were the two undominated strategies. Dabrafenib had an incremental effect of 0.35 QALYs and a cost-effectiveness ratio compared with dacarbazine of approximately $€ 250000$ per QALY gained. The combination vemurafenib in combination with cobimetinib have an incremental effect of 0.54 QALYs and incremental cost-effectiveness ratio compared with dabrafenib of about $€ 320000$ per QALY gained.

The results of PSA showed that dacarbazine is more likely to be cost effective than the alternatives for WTP values up to about $€ 223000$. The monotherapies showed a decreasing trend for further increasing WTP values, contrary to the combination strategies. When dacarbazine is excluded, the BRAF and MEK inhibitors in monotherapy had the highest probability of being cost-effective for the lowest WTP values and the combination strategies showing an increasing trend for very high WTP values.

\section{Scenario/sensitivity analyses}

The results of the PSA showed that in the BRAF and MEK group, the combination therapies were more effective (in form of QALY) but more expensive than monotherapies. For the immunotherapies, the new available treatment alternatives (nivolumab and pembrolizumab) was more effective but more costly than ipilimumab monotherapy and ipilimumab in combination with dacarbazine. The results showed that the combination of nivolumab and ipilimumab had about the same level effectiveness as nivolumab and pembrolizumab in monotherapy, but was clearly more expensive than the other immunotherapies alternatives (figure $3 \mathrm{~A}$ ).

In addition to the PSA, we performed some scenario analyses to test the uncertainty around some of the model assumptions and the input parameters.

\section{Alternative drug prices}

All the prices for the new drug interventions in our analysis are maximum pharmacy retail prices. To gain a clearer idea of cost-effectiveness, we conducted one-way sensitivity analyses to determine at what price each treatment would be considered cost-effective for a WTP of $€ 55850$.

The maximum pharmacy retail price would have to be reduced by approximately $79 \%$ for dabrafenib, $83 \%$ for trametinib, $84 \%$ for dabrafenib in combination with trametinib, $81 \%$ for vemurafenib, $84 \%$ for vemurafenib in combination with cobimetinib, $75 \%$ for ipilimumab, $63 \%$ for nivolumab and $64 \%$ for pembrolizumab. For the combination ipilimumab and dacarbazine, the drug cost of ipilimumab would need to be reduced by approximately $82 \%$. For the combination nivolumab and ipilimumab, a combined price reduction of about $76 \%$ would be necessary. Table 4 presents the results of the analysis. 
Table 4 Results of drug price scenario when the interventions refer to an incremental cost-effectiveness ratio of $€ 55850$ per QALY gained relative to dacarbazine

\begin{tabular}{|c|c|c|c|}
\hline Interventions & $\begin{array}{l}\text { Drug price at which } \\
\text { treatment is cost-effective } \\
\text { (WTP = €55850) }\end{array}$ & $\begin{array}{l}\text { Drug cost per month in } \\
\text { model }\end{array}$ & $\begin{array}{l}\text { Required rebate for cost- } \\
\text { effectiveness at WTP }=€ 55850 \\
(\%)\end{array}$ \\
\hline Dabrafenib & 1899 & 9040 & 79 \\
\hline Trametinib & 1675 & 9939 & 83 \\
\hline Dabrafenib+trametinib & 3127 & 18982 & 84 \\
\hline Ipilimumab* & 20328 & 82750 & 75 \\
\hline Ipilimumab†+Dacarbazine & 15190 & 82750 & 82 \\
\hline Nivolumab & 3574 & 9656 & 63 \\
\hline Nivolumab +lpilimumabł & 33508 & 138473 & 76 \\
\hline
\end{tabular}

*The ipilimumab cost is not per month, but the full treatment drug cost.

†Reduction applies to ipilimumab only.

$\ddagger$ The price reduction was estimated from the total cumulative drugs cost per patient, which combines the cost of ipilimumab and nivolumab.

WTP, willingness-to-pay.

Treatment until progression also for the PD-1 immunotherapies and different assumptions about treatment extension

We changed the structural assumption regarding treatment with nivolumab and pembrolizumab, from treatment independent of progression status and gradual reduction in the proportion being treated of those alive over time, to treatment in the progression free health state only, for 2 years. We also extended the treatment duration from 24 months to 36 months in two scenarios, assuming further treatment for $50 \%$ and $100 \%$ of those in the progression free health state at 2 years following treatment. The changes were only applied to the cost side of the model.

The results showed that costs are likely to be underestimated for nivolumab and pembrolizumab in monotherapy when the treatment duration is limited to 24 months and to the progression free health state only. The scenario that assumes that $100 \%$ of those in PFS at 24 months will be treated for another 12 months is the closest to our base case scenario. Our base case cost estimates for the immunotherapies are independent of the transition probability from PFS to progressed disease.

\section{Time-horizon (5 years vs 10 years)}

A time-horizon of 10 years was considered in the basecase analysis. We performed a scenario analysis where a time-horizon of 5 years was taken. The results showed that the time-horizon of 5 years influenced both costs and effects. However, the ranking of the interventions in the incremental analysis is unchanged when we change the time-horizon to 5 years instead of 10 years as in the base case (see online supplementary material 24). All other assumptions are the same as in the base case.
Quality of life weights

We performed a scenario analysis where we used the standard gamble weights from Beusterien et $a l^{73}$ for all interventions, which were 0.80 for PFS and 0.52 in progressed disease. Although some changes in the results can be observed, the choice of HRQoL weights and assumptions about different quality of life weights across the interventions does not seem to be decisive for the results of our cost-effectiveness analysis.

\section{DISCUSSION}

We have assessed the comparative effectiveness and cost-effectiveness of seven new drugs used for patients with advanced malignant melanoma in the Norwegian setting. The results are based on 17 RCTs. Our conclusions for the relative effectiveness of the included drugs or combinations of drugs rest on network meta-analyses using both direct and indirect evidence with dacarbazine as a common comparator. We ranked the different treatments in terms of their likelihood of leading to the best results for each clinical endpoint. The rankings were interpreted cautiously taking the quality of the evidence into account. The cost-utility analysis was based on a probabilistic discrete-time Markov cohort model.

When all drug interventions were compared, nivolumab, pembrolizumab, nivolumab in combination with ipilimumab, vemurafenib in combination with cobimetinib and dabrafenib in combination with trametinib had a higher probability of good performance for OS than monotherapies with ipilimumab or BRAF/MEK inhibitors.

Dabrafenib in combination with trametinib and vemurafenib in combination with cobimetinib had a higher probability of good performance for PFS than 
the immunotherapies, as well as the monotherapies of BRAF and MEK inhibitors. Ipilimumab had poorer PFS than monotherapy with the BRAF inhibitors as well as the BRAF inhibitors in combination with a MEK inhibitor.

Pembrolizumab and nivolumab seem to have a higher probability of fewer SAEs than the other treatment strategies, even though we could not establish any significant differences. However, since the quality of the evidence for SAEs were low or very low in most of our assessments (due to very few events and CrIs that included both benefit and harm), we have little confidence in these results. We have no results from network meta-analyses for HRQoL.

We find it difficult to separate the new immunotherapies (PD-1) nivolumab and pembrolizumab with respect to cost-effectiveness. They are both more effective and at the same time more costly compared with ipilimumab. Based on the cost-effectiveness results, we cannot argue that any of the BRAF or MEK inhibitor monotherapies (dabrafenib, vemurafenib, trametinib) should be preferred over another or that any BRAF/MEK combination (dabrafenib and trametinib or vemurafenib and cobimetinib) should be preferred over another. However, the combination therapies are more likely to give the highest quality adjusted life year gains.

None of the drugs investigated can be considered cost-effective at what has normally been considered a reasonable WTP in Norway. Drug price reductions (from official list prices) in the region of $63 \%-84 \%$ would be necessary for these drugs to be cost-effective at a WTP of $€ 55850$ per QALY.

\section{Comparison with other studies}

The Norwegian Medicines Agency (NOMA), CADTH, The Swedish Dental and Pharmaceutical Benefits Agency (TLV) and NICE, UK have performed single technology assessments on drugs used for metastatic melanoma. This has been done for dabrafenib versus dacarbazine ${ }^{1674}$ (NICE, TLV), dabrafenib in combination with trametinib versus dabrafenib ${ }^{15} 75$ (CADTH, TLV), dabrafenib in combination with trametinib versus vemurafenib ${ }^{15}$ $(\mathrm{CADTH})$, ipilimumab in combination with gp100 versus ipilimumab in previous treated patients ${ }^{17-19} 76$ (NMA, CADTH, NICE, TLV), ipilimumab in previous untreated patients (indirect comparisons to dacarbazine, vemurafenib and dabrafenib ${ }^{20} 7778$ (NICE, CADTH and TLV), trametinib versus dacarbazine ${ }^{21}$ (CADTH) and vemurafenib versus dacarbazine 222379 (NICE, CADTH and TLV). The results of these single technology assessments have been presented in online supplementary material 25 .

In our multiple technology assessment, we have included all the clinical trials used in these single technology assessments. None of these assessments has compared all the different new drugs for patients with advanced metastatic malignant melanoma relatively to each other. We have chosen not to explicitly compare our incremental cost-effectiveness results with the results of other published single technology assessments. Any such comparison would be highly dependent on how data on clinical effectiveness was used in the model, structural assumptions in the decision model and differences in cost data.

\section{Strengths and limitations of the study}

We found only two head to head comparison for the included drugs as monotherapies and five direct comparisons of combination treatment versus monotherapy. None of the included trials compared a BRAF inhibitor (dabrafenib or vemurafenib) head to head with a drug acting on the immune system. The best available comparisons are the indirect evidences via dacarbazine as a common comparator. All the interventions could be included in the network meta-analyses for OS, PFS and SAEs. HRQoL and SAEs are of importance for the patients. However, from the available literature we were not able to find data usable for our network meta-analysis for HRQoL, and the quality of the evidence for SAEs were low or very low in most of our assessments.

We only included RCTs and clinical endpoints were all defined and harmonised in their definitions across the trials.

The number of available interventions for patients with advanced malignant melanoma is evolving rapidly. Many of the interventions in this multiple technology assessment have just reached marketing authorisation in Norway, and the available evidence from RCTs is quite limited. Hence, clinical effectiveness data have the uncertainty that the majority of the evidence for the included comparisons were based on a single study. It cannot be ruled out that new evidence from future RCTs may have the potential to change the ranking of the interventions both with regard to effectiveness and cost-effectiveness.

Seven of the trials allowed patients in the control group to cross over to the intervention group after progression. The statistical analyses of the endpoint OS was based on the intention to treat population in all seven trials, that is, the analyses were done without taking the crossover into account. The assumption underlying the statistical analysis is that crossover does not alter mortality patterns seriously. However, we decided to include these data since the results for the intervention will not be favoured.

One of the trials that allowed crossover of patients from the control group to the intervention group did, however, present results from both analyses where participants who crossed over were censored at the time of crossover and analyses without censoring. ${ }^{61}$ They reported a slightly lower HR for death from the analysis with censoring at the time of crossover than from the analysis without censoring at the time of crossover. In our network meta-analyses we only included the data without censoring at time of crossover, since this was what we had for all the other trials.

Latimer $e t a l^{80}$ found that adjusting for switching resulted in lower HRs than those obtained from standard ITT analyses, however, CI are wide and results are sensitive to the assumptions associated with each adjustment method. 
We believe that the economic model distinguished the interventions fairly well with regard to costs and OS, but not so well with regard to HRQoL.

The use of different quality of life data resulted in different QALYs gained. However, the choice of HRQoL weights and assumptions about different quality of life weights across the interventions does not seem to be decisive for the results of our cost-effectiveness analysis.

Due to paucity in data, the network meta-analyses were not performed separately for the subgroups of patients with respect to those who were previously treated and those who were previously not treated. Therefore, we did not perform separate cost-effectiveness analyses for these two subgroups of patients. However, for cost-effectiveness, we performed three separate subgroup analyses: (1) including all interventions, (2) including only the BRAF and MEK inhibitors; monotherapies and combination therapies and (3) including only the immunotherapies.

There is lack of documentation regarding the longterm effect of the newer drugs. Further research could have the potential to change current estimates and consequently the health economic results.

\section{CONCLUSION}

- None of the drugs investigated can be considered cost-effective at what has normally been considered a reasonable WTP in Norway. Drug price reductions (from the official list prices) in the region of 63\%$84 \%$ would be necessary for these drugs to be cost-effective at a WTP of $€ 55850$ per QALY.

- Monotherapies with a PD-1 immune-checkpoint-inhibitor had a higher probability of good performance for OS than monotherapies with ipilimumab or BRAF/MEK inhibitors. The combination treatments had all similar levels of effectiveness to the PD-1 immune-checkpoint-inhibitors.

- PD-1 immune-checkpoint-inhibitors are more effective and more costly compared with ipilimumab in monotherapy. Nivolumab in combination with ipilimumab had higher costs and the same level of effectiveness as the PD-1 immune-checkpoint-inhibitors in monotherapy.

- BRAF/MEK inhibitor combinations (dabrafenib and trametinib or vemurafenib and cobimetinib) had both similar effectiveness and cost-effectiveness; however, the combination therapies are more likely to give higher quality adjusted life year gains than BRAF or MEK inhibitor monotherapies, but to a higher cost.

Acknowledgements We thank Einar Bjørner Torkilseng and Enrique Jimenez (health economists) for their assistance in the economic data modelling and research librarian Ingrid Harboe for her assistance in the literature searches.

Contributors EP carried out the clinical part and drafted the manuscript. VH carried out the economical part and drafted the manuscript. IS participated in the clinical part and commented on the manuscript. J0J performed the statistical analyses, co-developed the economic model and commented on the manuscript. MK was responsible for the project and participated in its planning, implementation and drafting of the manuscript.
Funding This work was supported by the Norwegian Knowledge Centre for the Health Services.

Competing interests None declared.

Provenance and peer review Not commissioned; externally peer reviewed.

Data sharing statement An extended version of this work is available at the web pages of the Norwegian Knowledge Centre for the Health Services: http://www. kunnskapssenteret.no/en/publications/A+health+technology+assessment+of+ the+new+drugs+for+inoperable+or+metastatic+malignant+melanoma+patients Appendices to the extended report including search strategy, tables of included and excluded studies, data analyses on crude and adjusted data and GRADE files are available in English.

Open Access This is an Open Access article distributed in accordance with the Creative Commons Attribution Non Commercial (CC BY-NC 4.0) license, which permits others to distribute, remix, adapt, build upon this work non-commercially, and license their derivative works on different terms, provided the original work is properly cited and the use is non-commercial. See: http://creativecommons.org/ licenses/by-nc/4.0/

(c) Article author(s) (or their employer(s) unless otherwise stated in the text of the article) 2017. All rights reserved. No commercial use is permitted unless otherwise expressly granted.

\section{REFERENCES}

1. Cancer Registry of Norway (Kreftregisteret). Nasjonalt kvalitetsregister for føflekkreft. rsrapport 2014. Oslo: Cancer Registry of Norway, 2014:41.

2. Skin Cancer Foundation. Melanoma: the skin cancer Foundation, 2015. http://www.skincancer.org/skin-cancer-information/melanoma

3. Dahl O, Lehne G, Baksaas I, et al; Malignt melanom, Kapittel $33 i$ Medikamentell kreftbehandling. cytostatikaboken 7. utgave 2009: Farmakologisk institutt, Det medisinske fakultet, Universitetet i Oslo, 2009. http://cytostatikaboken.moses.no/index.php?seks_id=7323\& path $=3466 \% 7$ C\&element=Kapittel

4. National Cancer Institute. Melanoma treatment-for health professionals $(P D Q \otimes)$ : National Institutes of Health, U.S. Department of Health and Human Services, 2015. http://www.cancer.gov/types/ skin/hp/melanoma-treatment-pdq.

5. Geisler J, Bachmann IM, Nyakas M, et al. Malignant melanoma-diagnosis, treatment and follow-up in Norway. Tidsskr Nor Laegeforen 2013;133:2154-9.

6. Cancer Registry of Norway (Kreftregisteret). Cancer in Norway 2013 Cancer incidence, mortality, survival and prevalence in Norway. Oslo: Cancer Registry of Norway, 2015.

7. Akslen $\mathrm{L}$, et al. Nasjonalt handlingsprogram med retningslinjer for diagnostikk, behandling og oppfølging av maligne melanomer. IS2238. Oslo: Helsedirektoratet, 2015:94 s.

8. Robert C, Schachter J, Long GV, et al. Pembrolizumab versus Ipilimumab in Advanced Melanoma. N Engl J Med 2015;372:2521-32.

9. Chapman PB, Hauschild A, Robert C, et al. Improved survival with vemurafenib in melanoma with BRAF V600E mutation. $N$ Engl J Med 2011;364:2507-16

10. Hauschild A, Grob JJ, Demidov LV, et al. Dabrafenib in BRAFmutated metastatic melanoma: a multicentre, open-label, phase 3 randomised controlled trial. Lancet 2012;380:358-65.

11. Larkin J, Ascierto PA, Dréno B, et al. Combined vemurafenib and cobimetinib in BRAF-mutated melanoma. N Engl J Med 2014;371:1867-76.

12. Robert C, Karaszewska B, Schachter J, et al. Improved overall survival in melanoma with combined dabrafenib and trametinib. $N$ Engl J Med 2015;372:30-9.

13. Robert C, Long GV, Brady B, et al. Nivolumab in previously untreated melanoma without BRAF mutation. N Engl J Med 2015;372:320-30.

14. Long GV, Stroyakovskiy D, Gogas $\mathrm{H}$, et al. Combined BRAF and MEK inhibition versus BRAF inhibition alone in melanoma. $N$ Engl $J$ Med 2014;371:1877-88.

15. Canadian Agency for Drugs and Technologies in Health (CADTH). Dabrafenib (Tafinlar) and trametinib (Mekinist) combo for metastatic melanoma - pERC final recommendation CADTH pan-Canadian oncology drug review, 2015:11.

16. National Institute for Health and Care Excellence (NICE). Dabrafenib for treating unresectable or metastatic BRAF V600 mutation-positive melanoma. : NICE technology appraisal guidance, 2014:321: 36.

17. Norwegian Medicines Agency (Statens legemiddelverk). Ipilimumab (Yervoy) til behandling av avansert malignt melanom hos voksne som 
er tidligere behandlet. Legemiddeløkonomisk vurdering. $2012 \mathrm{http}: / /$ www.legemiddelverket.no/Blaa_resept_og_pris/Helseoekonomiske\% 20rapporter/Documents/2012-2011/Yervoy_maligntmelanom_2012. pdf

18. Canadian Agency for Drugs and Technologies in Health (CADTH). Ipilimumab (Yervoy) for advanced melanoma - $p E R C$ final recommendation: CADTH pan-Canadian Oncology Drug Review, 2012:10.

19. National Institute for Health and Care Excellence (NICE). Ipilimumab for previously treated advanced (unresectable or metastatic) melanoma. : NICE technology appraisal guidance, 2012:268: 45.

20. National Institute for Health and Care Excellence (NICE). Ipilimumab for previously untreated advanced (unresectable or metastatic) melanoma. : NICE technology appraisal guidance, 2014:319: 52.

21. Canadian Agency for Drugs and Technologies in Health (CADTH). Trametinib (Mekinist) for metastatic melanoma - pERC final recommendation: CADHT pan-Canadian oncology drug review, 2013:10.

22. National Institute for Health and Care Excellence (NICE). Vemurafenib for treating locally advanced or metastatic BRAF V600 mutationpositive malignant melanoma, 2012.

23. Canadian Agency for Drugs and Technologies in Health (CADTH). Vemurafenib (Zelboraf) for advanced melanoma - $p E R C$ final recommendation: CADTH pan-Canadian oncology drug review, 2012:9.

24. Liberati A, Altman DG, Tetzlaff J, et al. The PRISMA statement for reporting systematic reviews and meta-analyses of studies that evaluate health care interventions: explanation and elaboration. $J$ Clin Epidemiol 2009;62:e1-e34.

25. Salanti G, Higgins JP, Ades AE, et al. Evaluation of networks of randomized trials. Stat Methods Med Res 2008;17:279-301.

26. Woods BS, Hawkins N, Scott DA. Network meta-analysis on the loghazard scale, combining count and hazard ratio statistics accounting for multi-arm trials: a tutorial. BMC Med Res Methodol 2010;10:54

27. Dias S, Welton NJ, Caldwell DM, et al. Checking consistency in mixed treatment comparison meta-analysis. Stat Med 2010;29:932-44.

28. Salanti G, Ades AE, loannidis JP. Graphical methods and numerical summaries for presenting results from multiple-treatment metaanalysis: an overview and tutorial. J Clin Epidemiol 2011;64:163-71.

29. Hamid O, Schmidt H, Nissan A, et al. A prospective phase II trial exploring the association between tumor microenvironment biomarkers and clinical activity of ipilimumab in advanced melanoma. J Transl Med 2011;9:204.

30. Robert C, Ribas A, Wolchok JD, et al. Anti-programmed-deathreceptor-1 treatment with pembrolizumab in ipilimumab-refractory advanced melanoma: a randomised dose-comparison cohort of a phase 1 trial. Lancet 2014;384:1109-17.

31. Higgins J, Green S. Cochrane Handbook for Systematic Reviews of Interventions: cochrane Collaboration, 2011.

32. GRADE working group. Grading of recommendations Assessment:

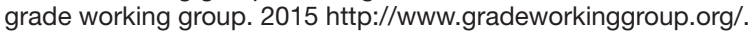

33. Puhan MA, Schünemann HJ, Murad MH, et al. A GRADE Working Group approach for rating the quality of treatment effect estimates from network meta-analysis. BMJ 2014;349:95630.

34. McMaster University. Guideline development tool (GDT): McMaster University and Evidence Prime Inc, 2015. http://www. guidelinedevelopment.org/.

35. Robert C, Thomas L, Bondarenko I, et al. Ipilimumab plus dacarbazine for previously untreated metastatic melanoma. N Engl J Med 2011;364:2517-26.

36. Maio M, Grob JJ, Aamdal S, et al. Five-year survival rates for treatment-naive patients with advanced melanoma who received ipilimumab plus dacarbazine in a phase III trial. $J$ Clin Oncol 2015;33:1191-6.

37. Briggs A, Claxton K, Sculpher MJ. Decision modelling for health economic evaluation. reprint 2011. ed. Oxford University Press: Oxford, 2006.

38. Postow MA, Chesney J, Pavlick AC, et al. Nivolumab and ipilimumab versus ipilimumab in untreated melanoma. Supplementary Appendix. N Engl J Med 2015;372:19.

39. Larkin J, Chiarion-Sileni V, Gonzalez R, et al. Combined Nivolumab and Ipilimumab or Monotherapy in untreated melanoma. N Engl J Med Overseas Ed 2015;373:23-34.

40. Wisløff T, Hagen G, Hamidi V, et al. Estimating QALY gains in applied studies: a review of cost-utility analyses published in 2010 . Pharmacoeconomics 2014;32:367-75.

41. Grob JJ, Amonkar MM, Karaszewska B, et al. Comparison of dabrafenib and trametinib combination therapy with vemurafenib monotherapy on health-related quality of life in patients with unresectable or metastatic cutaneous BRAF Val600-mutation- positive melanoma (COMBI-v): results of a phase 3, open-label, randomised trial. Lancet Oncol 2015;16:1389-98.

42. Köping-Höggård MSL, Lindgren $\mathrm{O}$, Kalin $\mathrm{Y}$, et al. Underlag for beslut i landstingen. Keytruda (pembrolizumab). TANDVÅRDS- $\mathrm{OCH}$ LÄKEMEDELSFÖRMÅNSVERKET2015:1-33.

43. Flaherty KT, Infante JR, Daud A, et al. Combined BRAF and MEK inhibition in melanoma with BRAF V600 mutations. N Engl J Med 2012;367:1694-703.

44. Ribas A, Puzanov I, Dummer R, et al. Pembrolizumab versus investigator-choice chemotherapy for ipilimumab-refractory melanoma (KEYNOTE-002): a randomised, controlled, phase 2 trial. Lancet Oncol 2015;16:908-18.

45. Hersh EM, O'Day SJ, Powderly J, et al. A phase II multicenter study of ipilimumab with or without dacarbazine in chemotherapynaïve patients with advanced melanoma. Invest New Drugs 2011;29:489-98.

46. Weber J, Hamid O, Amin A, et al. Randomized phase I pharmacokinetic study of ipilimumab with or without one of two different chemotherapy regimens in patients with untreated advanced melanoma. Cancer Immun 2013;13:7.

47. Hodi FS, O'Day SJ, McDermott DF, et al. Improved survival with ipilimumab in patients with metastatic melanoma. N Engl J Med 2010;363:711-23.

48. Larkin J, Chiarion-Sileni V, Gonzalez R, et al. Combined Nivolumab and Ipilimumab or Monotherapy in untreated melanoma. N Engl $J$ Med 2015;373:23-34.

49. Postow MA, Chesney J, Pavlick AC, et al. Nivolumab and ipilimumab versus ipilimumab in untreated melanoma. $N$ Engl $\mathrm{J}$ Med 2015;372:2006-17.

50. Revicki DA, van den Eertwegh AJ, Lorigan P, et al. Health related quality of life outcomes for unresectable stage III or IV melanoma patients receiving ipilimumab treatment. Health Qual Life Outcomes 2012;10:66

51. Maio M, Bondarenko I, Robert C, et al. Four-year survival update for metastatic melanoma (MM) patients (PTS) treated with ipilimumab (IPI) + dacarbazine (DTIC) on phase 3 study CA184-024. Asia Pac J Clin Oncol 2012;8:303.

52. Kotapati S, Francis S, Sherrill B. Health related quality of life (HRQL) of patients receiving ipilimumab with dacarbazine as first-line treatment for unresectable stage III/IV melanoma. Pigment cell melanoma res 2011;24:1037.

53. Long GV, Stroyakovskiy D, Gogas $\mathrm{H}$, et al. Dabrafenib and trametinib versus dabrafenib and placebo for Val600 BRAF-mutant melanoma: a multicentre, double-blind, phase 3 randomised controlled trial. Lancet 2015;386:444-51.

54. Weber JS, D'Angelo SP, Minor D, et al. Nivolumab versus chemotherapy in patients with advanced melanoma who progressed after anti-CTLA-4 treatment (CheckMate 037): a randomised, controlled, open-label, phase 3 trial. Lancet Oncol 2015:16:375-84.

55. Abernethy A, et.al. Effect of nivolumab (NIVO) in combination with ipilimumab (IPI) versus IPI alone on quality of life $(\mathrm{QoL})$ in patients (pts) with treatment-naïve advanced melanoma (MEL): results of a phase II study (CheckMate 069). J Clin Oncol 2015;33:abstr 9029.

56. Hauschild A, et.al. An update on BREAK-3, a phase III, randomized trial: dabrafenib (DAB) versus dacarbazine (DTIC) in patients with BRAF V600E-positive mutation metastatic melanoma (MM). J Clin Oncol 2013:1.

57. Hauschild A, Grobb J, Demidov L, et al. 1092pdan update on overall survival (OS) AND follow-on therapies IN break-3, a phase III, randomized trial: dabrafenib (D) VS. dacarbazine (DTIC) IN patients (PTS) WITH braf v600e mutation-positive metastatic melanoma (MM). Annals of Oncology 2014;25:iv378-iv93.

58. Grob J-J , et.al. A Landmark analysis of 3-year overall survival (OS) and Follow-On therapies in BREAK-3, a phase III, Randomized Trial: dabrafenib vs. Dacarbazine (DTIC) in patients (pts) with BRAF V600E Mutation-Positive metastatic melanoma. Poster: Society for Melanoma Research congress, 2014.

59. Grob JJ, Amonkar MM, Martin-Algarra S, et al. Patient perception of the benefit of a BRAF inhibitor in metastatic melanoma: qualityof-life analyses of the BREAK-3 study comparing dabrafenib with dacarbazine. Ann Oncol 2014;25:1428-36.

60. McArthur G, Hauschild A, Robert C, et al. Vemurafenlb improves Overall Survival compared to Dacarbazlne in Advanced BRAFV600E-mutated melanoma: updated Survival results from a phase III randomised, Open-label, Multicentre trial. Eur J Cancer 2011:47:14.

61. Hauschild A, McArthur GA, Robert C, et al. Vemurafenib improves overall survival compared to dacarbazine in advanced BRAFV600Emutated melanoma: an update from the phase III randomized, open-label, multicenter BRIM3 trial. Pigment cell \& melanoma res 2011;24:1021. 
62. Chapman PB, Hauschild A, Robert C, et al. Updated overall survival (OS) results for BRIM-3, a phase III randomized, openlabel, multicenter trial comparing BRAF inhibitor vemurafenib (vem) with dacarbazine (DTIC) in previously untreated patients with BRAFV600E-mutated melanoma. J Clin Oncol 2012;30.

63. McArthur GA, Chapman PB, Robert C, et al. Safety and efficacy of vemurafenib in BRAF(V600E) and BRAF(V600K) mutation-positive melanoma (BRIM-3): extended follow-up of a phase 3, randomised, open-label study. Lancet Oncol 2014;15:323-32.

64. Flaherty K, Daud A, Weber JS, et al. Updated overall survival (OS) for BRF113220, a phase 1-2 study of dabrafenib (D) alone versus combined dabrafenib and trametinib $(D+T)$ in pts with BRAF V600 mutation-positive (+) metastatic melanoma (MM). J Clin Oncol $2014 ; 1$.

65. Daud A , et.al. Updated overall survival (OS) results for BRF113220, a phase I-II study of dabrafenib alone versus combined dabrafenib and trametinib in patients with BRAF V600 metastatic melanoma (MM). $J$ Clin Oncol / 2015;33:9036.

66. Schadendorf D, Amonkar MM, Stroyakovskiy D, et al. Healthrelated quality of life impact in a randomised phase III study of the combination of dabrafenib and trametinib versus dabrafenib monotherapy in patients with BRAF V600 metastatic melanoma. Eur $J$ Cancer 2015;51:833-40.

67. Larkin J , et.al. Update of progression-free survival (PFS) and correlative biomarker analysis from coBRIM: phase III study of cobimetinib (cobi) plus vemurafenib (vem) in advanced BRAFmutated melanoma. J Clin Oncol 2015;33:abstr 9006.

68. Dréno B , et al. Quality- of-life (QOL) assessment in patients (pts) with metastatic melanoma receiving vemurafenib $(V)$ and cobimetinib (C). J Clin Oncol 2015;33:abstr 9021.

69. Flaherty KT, Robert C, Hersey P, et al. Improved survival with MEK inhibition in BRAF-mutated melanoma. N Engl J Med 2012;367:107-14.
70. Schadendorf $D$, et.al. Overall survival (OS) update on METRIC (NCT01245062), a randomized phase 3 study to assess efficacy of trametinib (T) compared with chemotherapy (C) in patients (pts) with BRAFV600E/K mutationpositive (+) advanced or metastatic melanoma (MM). Poster: Society for Melanoma Research Congress (SMR), 2013.

71. Schadendorf D, Amonkar MM, Milhem M, et al. Functional and symptom impact of trametinib versus chemotherapy in BRAF V600E advanced or metastatic melanoma: quality-of-life analyses of the METRIC study. Ann Oncol 2014;25:700-6.

72. Weber JS, D'Angelo SP, Minor D, et al. Nivolumab versus chemotherapy in patients with advanced melanoma who progressed after anti-CTLA-4 treatment (CheckMate 037): a randomised, controlled, open-label, phase 3 trial. Lancet Oncol 2015;16:375-84.

73. Beusterien KM, Szabo SM, Kotapati S, et al. Societal preference values for advanced melanoma health states in the United Kingdom and Australia. Br J Cancer 2009;101:387-9.

74. The Swedish Dental and Pharmaceutical Benefits Agency (TLV). Tafinlar for advanced melanoma, 2014.

75. The Swedish Dental and Pharmaceutical Benefits Agency (TLV). Mikinist for advanced melanoma, 2016.

76. The Swedish Dental and Pharmaceutical Benefits Agency (TLV). Ipilimumab for Advanced Melanoma, 2012.

77. Canadian Agency for Drugs and Technologies in Health (CADTH). Ipilimumab for Advanced Melanoma, 2012:04.

78. The Swedish Dental and Pharmaceutical Benefits Agency (TLV). Ipilimumab for Advanced Melanoma, firstline treatment, 2014.

79. The Swedish Dental and Pharmaceutical Benefits Agency (TLV). Zelboraf for Advanced Melanoma, 2012.

80. Latimer NR, Bell H, Abrams KR, et al. Adjusting for treatment switching in the METRIC study shows further improved overall survival with trametinib compared with chemotherapy. Cancer Med 2016;5:806-15 